\title{
EXPLOITING REFERENCE INTERACTION IN RESOLVING TEMPORAL REFERENCE
}

\author{
Kohji DOHSAKA \\ NTT Basic Research Laboratories \\ 3-1 Morinosato-Wakamiya, Atugi-shi, Kanagawa, 243-01 Japan \\ dohsaka@atom.ntt.jp
}

\begin{abstract}
This paper provides an account of the role that the interaction between nominal and temporal reference plays in resolving temporal reference. Fixploiting this interaction in resolving temporal reference clarifies how the process of resolving nominal reference interacts with the process of resolving temporal refercnce, and how a restricted set of world knowledge contributes to resolving temporal reforence.
\end{abstract}

\section{INTRODUCTION}

Resolving reference or anaphora is of great interest in computational linguistics and formal semantics. Research on reference began with the development of models to account for nominal reference brought about by the usage of nominal expressions such as definite noun phrases and pronominals (Grosz, Joshi and Weinstein 1983; Kameyama 1986). Recently a number of researchers have indicated that temporal expressions such as tense morphemes and temporal adverbials can be regarded as referring expressions and have proposed models for temporal reference resolution (Hinrichs 1986; Hwang and Schubert 1992; Kameyama, Passonneall and Poesio 1993; Lascarides 1992; Partee 1984; Song and Cohen 1991; Webber 1988).

Sentences in a dialogue describe eventualities ${ }^{1}$ and introduce them into the context. The time of an eventuality described by a sentence is interpreted as temporally related to the times of other eventualities that were introduced into the context earlier in the dialoguc. Temporal expressions are regarded as referring expressions since they convey information about the time of an eventuality, which is interpreted relative to the times of other eventualities in the context. Resolving temporal reference means determining what temporal relationships exist between an eventuality described by a sentence of a dialogue and cventualities that have been introduced into the context earlier in the dialogue. "2 Resolving temporal reference is requisite to many tasks, such as designing a natural language interface to a planning system in which temporal information is crucial (Crouch and Pullman

\footnotetext{
${ }^{2}$ An eventuality is the general term for an event, process or state, due to Bach (1986).

${ }^{2}$ The chief concern here is temporal reference to intersentential context. 'Thus this paper does not address the problem of determining what temporal relationships an eventuality described by a clause of a sentence has with eventualities described by other clauses of the same sentence. This problem is covered by Brent (1990), Ifwnng et al. (1992), and Reichenbach (1947).
}

$1993)$

To understand the notion of temporal reference, consider the following dialogue, (d1). ${ }^{3}$ Assume that dialogue participants, Bill and John, mutually know that John received an e-mail message from Mary.

Dialogue (d1)

(s1) Bill: Mary karano mail wa mot-teiru-yone? from mail TOPhave-PRESENT You have the mail from Mary, don't you?

(s2) John: Keshi-ta-kedo. delete-PAS' $I$ deleted it.

(s3) Bill: Hontoni! really Really!

(s4) John: Sochira niwa tensoushi-ta-hazuda-yo. you DA' forward-PAST-sure. I'm sure that I forwarded it to you.

(s5) John: Sochira no mail box ni at-ta-desho? you GEN mail box in be-PAST. It was in your mail box, wasn't it?

Sentence (s2) describes an eventuality of deleting, $E_{d}$, and sentence (s4) describes an eventuality of forwarding, $E_{f}$. Both eventualities are past ones, since the matrix verbs, "tensousuru (forward)" and "kesu (delete)", "describe momentary acts and are accompanied with the past-tense morpheme " $\mathrm{ta}$ ". Although the tense morphemes of sentences convey information about the times of eventualities described by the sentences, the context also imposes restrictions on the times of the eventualities. In dialogue (d1), the time of $E_{f}$ described by (s4) is interpreted as relative to the time of $E_{d}$ in the context: i.e. the time of $E_{f}$ is before the time of $E_{d}$. In this sense, the time of $E_{d}$ is referred to in uttering sentence (s4).

Existing models of temporal reference account for the parallel between temporal and nominal reference. However, as I will state in sec. 2, existing models fail to explain the interaction between temporal reference and nominal reference (reference interaction for short). Reference interaction is a phenomenon in which the process of resolving nominal reference in-

\footnotetext{
${ }^{3}$ In exhibiting a Japanese dialogue, English words such as "Mary" and "mail" are used only for easy comprehensibility. In addition, sentence-final forms such as "yone", "kedo", "yo", and "desho" indicate mental states of the speaker that are unrelated to the subject of this paper.

4 "tensoushi-" and "keshi-" in the dialogue are inflections of the verbs "tensousuru" and "kesu" respectively.
} 
teracts with the process of resolving temporal referonce, when an uttcrance involves temporal and noninal reference at the same time. Due to an insufficient account of the reference interaction, existing models cannot show how the process of resolving nominal reference affects the process of resolving temporal reference.

'The chief concern of this paper is to describe the role that the reference interaction plays in resolving temporal reference and demonstrate that the reference interaction serves to clarify low a restricted set of world knowledge contributes to the resolution process. I focus on sentences with past-tense morphemes in Japanese dialogues. Previous work also used pasttense sentences as a touchstone to show the validity of a model (IIinrichs 1986; Kameyana et al. 1993; I ascarides 1992; Partee 1984; Webber 1988). As I will state in sec. 2.2 , the reference interaction is not a domestic phenomenon in Japarese clialogues.

In sec, 2, I argue that existing models do not ac. count for the reference interaction, that the reference interaction plays an important role in alt account of how temporal reference is resolved, and that exploiting the reference interaction clarifies how a restricted set of world knowledge serves to resolve temporal reference. In sec. 3, a framework is presented, within which the reference interaction is exploited in resolving temporal reference. In sec. 4, I demonstrate how the franework works. In sec, 5, I summarize the claims of this paper and describe future work.

\section{PROBLEMS WITH EXISTING MODELS}

\subsection{Approaches in existing models}

In cxisting models, when the time, $T^{\prime}$, of an eventuality described by a sentence of a dialogue is given, the problem of resolving temporal reference is divided into (i) that of identifying the time, $T_{t j}$, of an eventuality that has been introduced into the context, and (ii) that of determining what temporal relationship holds between $T$ ' and ' $T$ if.

The notion of temporal focus is used to solve prob. lem (i). 'The notion was introduced by Webber (1988). Dialogue participants pay attention to the time of an eventuality that is in the temporal focus. 'The time, $T$, of an eventuality described by the current sentence is interpreted according to the time, $T_{t f}$, in the temporal focus. Ixisting models that apply to intersentential context use the notion of temporal focus (II wang et al. 1992; Song ct al. 1991). Kaneyama el al. (1993) refined the notion of temporal focus and proposed the notion of temporal centering. In formal semantics, models of temporal anaphora based on disconrse representation theory have bees proposed (Minrichs 1986; Partee 1981). 'These models concentrate on the tenso interpretation of adjacent sentences in a narrative dis course. 'Thus, the time of an eventuality described by a sentence immediately preceding the current sentence can be regarded as being in the temporal focus.

there are two approaches to problem (ii): (ii-a) an approach based on linguistic cues such as tenso morphemes, the aspectual class of verbs, and the descriptive order of eventualities in a dialogue; (ii-b) an approach based on world knowledge of the causal re lationships between eventualities.

With regard to approach (ii-a), the following default interpretation rules (Iinrichs 1986; Kameyama et al. 1993; Lascarides 1992; Partee 1984; Webber 1988) have been used."

\section{(Default interpretation rules)}

Provided that an eventuality, $h$, is described by the current sentence, the time of an eventuality, $b_{t f}$, is in the temporal focus, and both $k$ and $H_{t f}$ are past cventualitios, then

(R1) The time of $E^{\prime}$ is after the time of $E_{t f}$ if both $F$ and $l_{t f}$ are non-stative,

(1R2) The time of $E$ contains the time of $l^{\prime} t f$ if $H '$ is stative and $E_{i f}^{\prime}$ is non-stative.

These rules are termed default interpretation rules since they are utilized when world knowledge of cansality is not available.

In approach (ii-b), the temporal relationship betwoen $b^{\prime}$ and $l_{t f}$ is determined according to world knowledge of the cansal relationships between eventualities. Jascarides (1992) presented a model based on a theory of defcasible inference for integrating world knowledge of causality with the process of determining the temporal and causal relationships between eventualities mentioned in a discourse. 'This approach was also adopted in in model proposed by Kameyama $c t a l$. (1993). In these models, approaches (ii-a) and (ii-b) are unified within a single framework. Let me call the interpretation based on approach (ii-a) the default interprotation, and the interpretation based on approach (ii-b) the knowledge-based interpretation. The knowledge-based interpretation is preferred over the default interpretation when they disagreo.

Jet me explain how existing models work by using dialogue (d1) as an example. I focus on how (st) is interpreted, assuming that the time of $E_{d}$ is in the temporal focus. Default interpretation rulc (R1) says that the time of $E_{f}^{\prime}$ must be after the time of $H_{d}^{\prime}$ since both eventualities are non-stative, past eventualities. However, the relevant interpretation is that $E_{f}$ temporally precedes $H_{d}^{\prime}$. 'Thus, the default interpretation does not coincide with the relevant interpretation. In this case, existing models resort to world knowledge of the causal relationships between $E_{d}$ and $E_{f}$. How. ever, existing models do not, clarify what kind of world knowledge is used (Kameyama et al. 1993; Lascarides 1992). In this sense, the world knowledge used in existing models is unrestricted. I agree with such an approach in that various causal relationships are in. volved in the process of resolving reference. However, it is desirable to find a restricted set of world knowladge and avoid resorting directly to the entire set of

\footnotetext{
${ }^{\mathrm{B}}$ Note that the time of a stative eventuality is usually not regarded as being in the temporal focus.
} 
world knowledge of causality since such knowledge is enormous.

Consequently, existing models exploit the notion of temporal focus or temporal center, which serves only to show that temporal reference is accounted for in the same way as nominal reference. However, existing models do not explain how the process of resolving nominal reference affects the process of resolving temporal reference and assume that the entire set of knowledge of causality can be used.

\subsection{Reference interaction}

In this section, I argue that the notion of reference interaction provides an account of the temporal reference in (s4), which existing approaches ignore. Moreover, a restricted set of world knowledge can be used to explain the reference interaction.

Sentence (s1) introduces an individual ${ }^{6}, M$, which is an e-mail message. Sentence (s4) involves nominal reference to $M$ : the object of "tensousuru (forward)" is zero-pronominalized and refers to $M$. Moreover, sentence ( $s 4$ ) involves the interaction between nominal and temporal reference. That is to say, what the object being forwarded is affects what the time of forwarding is. I will explain this below.

When someone forwards something to someone else, the time of forwarding must be during the time when the object being forwarded exists. In other words, the time of forwarding depends on what the object being forwarded is. Moreover, when someone deletes something, the time when the object being deleted exists must be before the time of deleting. This kind of world knowledge can be regarded as knowledge of temporal relationships between eventualities and the existential status of individuals. Judging from such knowledge, the eventuality of forwarding, $F_{f}$, cannot temporally follows the eventuality of deleting, $E_{d}$. This is because both the object of "kesu (delete)" and the object of "tensousuru (forward)" refer to $M$, so the time of $E_{f}$ must be during the time when $M$ exists and the time when $M$ exists must be before the time of $F_{d}$. 'The interpretation consistent with such knowledge is that $E_{f}$ temporally precedes $E_{d}$. Consequently, identifying the referent of the zero-pronominalized object of $E_{f}$ serves to resolve temporal reference, and the knowledge of temporal relationships between eventualities and the existential status of individuals can be used to explain the reference interaction. ${ }^{7}$

Morcover, knowledge of the temporal relationships between eventualities and the existential status of individuals can be regarded as a restricted set of world knowledge of causality. It is restricted because given an eventuality, $E$, we have only to allow for the individuals that constitute $E$ and do not have to allow

\footnotetext{
"Fere, "individual" is used as a term for a single person or thing.

Tn this paper, only nominal refcrence to individuals is allowed for.
}

for all eventualities that can be causally related to $E$. Exploiting the reference interaction clarifies how such a restricted set of world knowledge contributes to resolving temporal reference. When such restricted world knowledge is sufficient to resolve temporal reference, immediate recourse to the entire set of world knowledge of causality can be avoided. Note that such knowledge is not selected arbitrarily. It is the knowledge that is necessary for explaining the reference interaction.

Next I review two existing proposals that are related to the argument above. First, Hwang et al. (1992) argued that resolving temporal reference requires plausible inference that can interact with various processes such as resolution of anaphora, introduction of new individuals and identification of spatial and temporal frames. They also argue that the plausible inference has to rely on world knowledge such as that one normally would not buy broken things (IIwang et al. 1992: p.239). Their argument agrees with the approach presented here. They did not, however, present a concrete model to support the argument.

Second, Webber and Baldwin (1992) discussed the integration of two independent mechanisms for context-change by entity introduction and by event simulation. The idea of integrating these contextchange mechanisms and that of exploiting the reference interaction in resolving reference share the view that the relationships between eventualities and the existential status of entities or individuals serve in interpreting referring expressions. They, however, focused on interpreting nominal reference made by the use of definite nouns, rather than on the problem of resolving temporal reference by exploiting the reference interaction, which is the chief concern of this paper.

Finally, it is casy to sec that the reference interaction also occurs in an English dialogue. In an English counterpart of $(\mathrm{d} 1)$, the pronoun "it" is used the same way as a Japanese zero pronoun is used. Likewise, the restricted knowledge stated above is common to Japanese and Lnglish.

\section{A FRAMEWORK}

A framework is presented, within which the reference interaction is exploited in resolving temporal reference. First, in sec. 3.1, the descriptive device is shown. Next, in sec. 3.2 , the process of resolving temporal refcrence is described.

\subsection{A descriptive device}

In a dialogue, sentences convey information about the speakers' mental attitudes toward eventualities. This framework does not concern the mental attitudes, but focuses on the eventualities described in the sentences. An eventuality is written as a variant of the Davidsonian represcntation (Davidson 1980; Vlach 1993). For cxample, the informational content of the eventuality of forwarding described by (s4) is written as follows. 
(r1) T'ype $\left(* \varepsilon_{f 1}\right.$, t'orward $) \&$

Agent $\left(* e_{f 1}, * a g e n t_{1}\right) \& \operatorname{Recp}\left(* c_{f 1}, * r e c p_{1}\right) \&$

Object $\left(* c_{f 1}, * o b j_{1}\right) \& l^{\prime} I^{\prime m} \operatorname{loc}\left(* c_{f 1}, * t_{f 1}\right) \&$

NonStative $\left(* e_{j 1}\right) \&$ Hearer $\left(* r^{2} c p_{1}\right) \&$.

T'Rel (Before, $\left.* t_{f 1}, * t_{s p 1}\right) \&$

T'Rel $\left(* r e l_{1}, * t_{f 1}, * t_{f o c u s}\right) \&$

Specch't'ime (*t $\left.t_{s p 1}\right)$ \&'l'empFrocus $\left(* t_{\text {focus } 1}\right)$.

Symbols starting with a capital letter represent constants and symbols starting with the character ' $*$ ', represent variables. Symbol ' $\&$ ' is an operator for conjunction.

Type $(H, T)$ means that eventuality $F$ ' is classified as a type $T$. Representation (rl) specifies the eventuality, $* e_{j}$, that $* a g e n t_{1}$ forwards $* o b j_{1}$ to $* r e c p_{1}$ at time $* t_{f 1}$. 'The agent and the object of forwarding are represented as variables, since they are zeropronominalized. Hearer $\left(l^{\prime}\right)$ means that $l^{\prime}$ is the hearer. In the above, the recipient of forwarding is identified with the hearer since it is specified by the pronoun "sochira", designating the hearer. In addi tion, eventuality $* e_{f 1}$ is non-stative

Spechtime $(T)$ means that tine $T$ is the specch time, Templ'ocus( $I$ ') means that tine ' $T$ ' is in tho tenporal focus, and $T$ 'Rel $\left(R, T_{1}, T_{2}\right)$ means that temporal relationship $l 2$ holds between time $t_{1}$ and $T_{2}$ In this framework, temporal relationships are represented based on temporal logic proposed by Allen (1983) and times are treated as temporal intervals. In the above, time $* t_{f 1}$ is before speech timo $* t_{s p 1}$ since * $t_{f 1}$ is specified by the past-tense morpheme "tai". Time $* t_{\text {focus } 1}$ is in the temporal focus. 'Temporal relationship between $* t_{f 1}$ and $* l_{f o c u s s}$ is represented as a variable, $* r e l_{1}$. Resolving temporal relerence means determining a relevant temporal relationship betweeen $* t_{f 1}$ and $* t_{f o c u s 1}$.

Knowledge of the temporal relationship between an eventuality and the existential status of individuals is represented as a triplet $\langle I R,\{I R, \ldots\}, T R\rangle$, where $I R$ is a representation for the eventuality, $\{l R, \ldots\}$ is a set of representations of the existen tial status of the individuals, and ' $T R$ is the temporal relationship between them. 'This framework concerns the existential status of individuals such that an individual cxists at a cortain space-tine location of the physical world. lior exanple, knowledge about an eventuality of forwarding is written as follows.

$$
\begin{aligned}
& (\mathrm{r} 2)<\mathrm{HR}_{f},\left\{\mathrm{IR}_{f_{a}},\left[\mathrm{R}_{f b}\right\}, ' \mathrm{I} \mathrm{R}_{f}\right\rangle \text {, where } \\
& H_{f} \stackrel{\text { def }}{=} T y p e\left(* e_{f}, F^{\prime} \text { rwuard }\right) \& \\
& \text { Agent }\left(* c_{f}, * \text { (lgonl }\right) \& \\
& \operatorname{Recp}\left(* \sigma_{f}, * \operatorname{rec} p\right) \& \\
& \text { Object }\left(* e_{f}, * a b j\right) \& \\
& \text { I'ime }\left(* c_{f}, * t_{f}\right) \text {, } \\
& I R_{f a} \stackrel{\text { def }}{=} T y p e\left(* e_{o b j}, L^{\prime} x i s t\right) \& \text {. } \\
& \text { Object }\left(* e_{o b j}, * o b j\right) \& \\
& \text { loc }\left(* \epsilon_{o b j}, * l_{o b j}\right) \& \\
& \text { Ouner }\left(* l_{o b j}, * \text { agent }\right) \& \\
& \text { I'ime }\left(* e_{o b j}, * t_{o b j}\right) \text {, }
\end{aligned}
$$

$$
\begin{aligned}
& \left.I R_{f b} \stackrel{\text { def }}{=} \text { 'I'ypc(*copy }, L^{\prime} x i s t\right) \& \\
& \operatorname{Objcct}\left(* e_{\operatorname{cop} y}, * \operatorname{cop} y\right) \& \text {. } \\
& \text { CopyOf }(* \circ o p y, * o b j) \& \\
& \text { Loc }\left(* c_{\text {copy }}, * I_{\text {cop } y}\right) \& \text {. } \\
& \text { Ouner }\left(* l_{\text {copy }}, * \text { *ecp }\right) \& \\
& \text { Time (*e copy } \left., * t_{\text {copy }}\right) \text {, } \\
& \left.T R_{f} \stackrel{\text { def }}{=} T \text { Rel }(I) \text { uring }, * t_{f}, * t_{o b j}\right) \& \\
& \text { Thell(Before, } \left.* t_{f}, * t_{\text {copy }}\right) \text {. }
\end{aligned}
$$

Loc $(* x, * l)$ moans that individual $* x$ exists at lo. cation $* l$. In this dialogue domain, the location where an e-mail message exists is a mail box. Owner $(* l, * p)$ means that person $* p$ owns location $* l$. I assume here that the owner of a mail box is uniquely identified.

In the above, $I_{f a}$ specifies the existential status of $* o b j$, which is the object being forwarded, and says that $*_{o b j} j$ exists at time $* t_{o b j}$ and at location $* l_{o b j}$, which the agent of forwarding owns. $l h_{f b}$ specifies the existential status of *copy, which is a copy of $* o b j$ and is generated by forwarding *obj. The object that the recipient of forwarding receives is not identified with *obj because of domestic constraints concerning an e-mail system. It fo says that $*$ copy exists at time $* t_{\text {copy }}$ and at location $* l_{c o p y}$, which the recipient of forwarding owns. $7 l_{f}$ says that the time of forwarding, $* t_{f}$, must be during the time, $* t_{o b j}$, when $*$ obj exists, and that the time of forwarding, *tf must be before the time, *tcopy, when $*$ copy exists.

Knowledge about an eventuality of deleting is writton as follows.

$$
\begin{aligned}
& \text { (n3) }<\mathbb{H R}_{d,},\left\{\mathrm{IR}_{d}\right\}, \Gamma R_{d l}>\text {, where } \\
& \text { I. } R_{d} \stackrel{\text { def }}{=} T \text { ype }\left(* e_{d}, \text { Delcte }\right) \& \\
& \text { Agent }\left(* e_{d}, * a g e n t\right) \& \\
& \text { Object }\left(* e_{d}, * o b j\right) \& \text {. } \\
& \text { Time }\left(* e_{s l}, * l_{d}\right) \text {, } \\
& I R_{d} \stackrel{\text { dst }}{=} \text { Type }\left(* e_{o b j}, \text { lixist }\right) \& \\
& \text { Object }\left(* e_{o b j}, * o b j\right) \& \\
& \text { loc }\left(* e_{o b j}, * I_{o b j}\right) \& \text {. } \\
& \text { Owner }\left(* l_{o b j}, * \text { agent }\right) \& \text {. } \\
& \text { T'ime }\left(* e_{o b j}, * t_{o b j}\right) \text {, } \\
& T h_{d l} \stackrel{\text { sef }}{=} T \text { Rel }\left(\text { Meet }, * \ell_{o b j}, * t_{d l}\right) \text {. }
\end{aligned}
$$

$I T_{d}$ says that the object being deleted, $*$ obj, exists at time $* l_{d}$ ancl at location $* l_{o b j}$ owned by the agent of deleting, *agent. $T R_{d}$ says that the time when *obj exists must be immediately before the time of deleting.

\subsection{Resolving temporal reference}

'This framework assumes that at representation of an eventuality described by a sentence is given. In the representation, pronominalized individuals and indeterminate temporal relationships are represented as variables such as *agent 1 and $* r e l_{1}$ in $(\mathrm{r} l)$. When the representation of the eventuality described by the current sentence is given, representations of the existential status of individuals and temporal relationships between the eventuality and the existential status of individuals are derived by using knowledge such as 
(r2) and (r3). These representations are interpreted within the context as described below. In the interpretation process, appropriate constants are substituted for variables. After the interpretation process, the representations are introduced into the context. Thus, the context includes representations of eventualities, the existential status of individuals, and temporal relationships among the eventualities and the existential status of individuals that have been mentioned in a dialogue. In addition, it includes representations that show who is the speaker, who is the hearer, what is the speech time, and what is the time in the temporal focus.

Let RSet be a set of representations of an eventuality, $E$, described by a sentence, the existential status of individuals mentioned in $E$, and temporal relationships between them. The interpretation process is as follows.

(I1) $\Lambda$ representation, $R$, in $R$ set is unified with a representation, $R_{c}$, in the context, if possible. Through unification, constants in $R_{c}$ are substituted for variables in $R$.

(I2) An indeterminate temporal relationship between the time of eventuality $E$ and the time in the temporal focus is identified with the relevant temporal relation according to default interpretation rules and transitive and reflexive laws governing temporal relationships.

(13) Constants are generated and substituted for variables that cannot be identified in steps (I1) and (I2) of this process.

(14) Representations in Rset are added to the context.

Some nominal reference is resolved in (I1), although this paper does not go into how nominal reference is resolved.

In (I2), temporal reference is resolved. The interpretation by transitive and reflexive laws governing temporal relationships is preferred over the interpretation by default interpretation rules.

This framework uses the default interpretation rules (R1) and (R2), which are used in existing models. The default interpretation rules (R1) and (R2) are represented as the following theorems, (14) and (r5) respectively. ${ }^{8}$

(r4) Time $\left(* e_{i}, * t_{i}\right) \& N$ NonStative $\left(* e_{i}\right) \&$ Time $\left(* e_{t f}, * t_{t f}\right) \& N$ onStative $\left(* e_{t f}\right) \&$ TempFocus $\left(* t_{t f}\right) \supset T \operatorname{Rel}\left(\right.$ After, $\left.* t_{i}, * t_{t f}\right)$.

(r5) Time $\left(* e_{i}, * t_{i}\right) \&$ Stative $\left(* e_{i}\right) \&$ Time $\left(* e_{t f}, * t_{t f}\right) \&$ NonStative $\left(* e_{t f}\right) \&$ TempFocus $\left(* t_{t f}\right) \supset$ TRel(Contains, $\left.* t_{i}, * t_{t f}\right)$

In the above, $R_{1} \supset R_{2}$ means that $R_{1}$ implies $R_{2}$.

The transitive and reflexive laws governing temporal relationships are also represented as theorems. For brevity's sake, I will not present all the laws. Allen (1983) presents a exhaustive list of transitive

\footnotetext{
${ }^{8}$ Here I ignore conditions where the eventualities at stake are past ones.
}

laws governing temporal relationships. 'The following theorems are sufficient for dealing with dialogue (d1).

(r6) Trel(Juring, *x,*z)\&T'rel(Meet, *z,*y) つ Trel( Before, $* x, * y)$.

(r7) Trel(Before, $* x, * y) \supset$ Trel(After, $* y, * x)$.

(18) Trel(During, $* x, * y) \supset$ Trel(Contains, $* y, * x)$.

The interpretation by the transitive and reflexive laws governing temporal relationships can be regarded as a kind of of knowledge-based interpretation as described in sec. 2.1, although only a restricted set of world knowledge is used in this framework. As demonstrated in sec. 4 , the interaction between references to individuals and times plays an important role in rosolving temporal reference according to the transitive and reflexive laws governing temporal relationships.

\section{EXAMPLES}

This section demonstrates how the framework works by using sentence ( $(s 4)$ and ( $(55)$ as examples. First, consider the interpretation of ( $\mathrm{s} 4)$ under the context established by $(\mathrm{s} 2)$. Sentence ( $\mathrm{s} 2)$ introduces an eventuality of deleting, $E_{d}$, into the context. The eventuality is represented as follows.

(r9) Type $\left(E_{d}\right.$, Delete $) \&$

$$
\begin{aligned}
& \text { Agent }\left(E_{d}, J \text { ohn }\right) \& O b j e c t\left(\omega_{d}, M\right) \& \\
& \text { Time }\left(E_{d}, T_{d}\right) \& N \text { onStative }\left(E_{d}\right) \&
\end{aligned}
$$$$
\text { Before }\left(F_{d}, T_{s p}\right) \text {. }
$$

In (r9), $M$ represents an individual, which is an e-mail message, $T_{d}$ represents the time of $E_{d}$, and $T_{s p}$ represents the time when sentence $\left(s^{2}\right)$ is uttered. ${ }^{g}$

By using knowledge ( $\mathrm{r} 3$ ), the existential status, $E_{m}$, of $\mathrm{M}$ and the temporal relationship between $E_{d}$ and $E_{m}$ are derived and introduced into the context. They are written as follows.

$$
\begin{aligned}
& \text { (r10) Type }\left(L_{m}, \text { Exist }\right) \& \\
& \text { Object }\left(E_{m}, M\right) \& \operatorname{Ioc}\left(E_{m}, L_{m}\right) \& \\
& \text { Owner }\left(L_{m}, J \text { John }\right) \& T \text { Iime }\left(H_{m}, I_{m}\right) \text {. } \\
& \text { (r11)TRel(Meet } \left., T_{m}, T_{d}\right) \text {. }
\end{aligned}
$$

$T_{m}$ represents the time when the e-mail message $M$ exists and $L_{m}$ represents the location of $M$.

The context also includes the following representation when (s4) is interpreted.

(r12) Speaker(John)\&Hearer (Bill).

(r13) SpeechTime $\left(T_{s p}\right) \& T$ TempFocus $\left(T_{d}\right)$.

Namely, I assume that the time of deleting, $T_{d}$, is in the temporal focus when (s4) is interpreted.

Now, let me explain how sentence (s4) is interpreted under the above context. Sentence (s4) describes an eventuality of forwarding, $* e_{f 1}$, which is written as representation ( $\mathrm{r} 1$ ) described in scc. 3.1. Likewise, by using knowledge ( $r 2$ ), the following representations are derived.

(r14) Type (*eobj1, Exist)\&

$$
\begin{aligned}
& \text { Object }\left(* e_{o b j 1}, * o b j_{1}\right) \& \operatorname{Loc}\left(* e_{o b j 1}, * l_{o b j 1}\right) \& \\
& \text { Owner }\left(* l_{o b j 1}, * a g e n t_{1}\right) \& \operatorname{Time}\left(* e_{o b j 1}, * l_{o b j 1}\right) .
\end{aligned}
$$

\footnotetext{
${ }^{9} \mathrm{I}$ assume that all sentences of a dialogue are uttered in the same temporal interval, $T_{s p}^{\prime}$.
} 
(r15)Type $\left(* e_{\text {copy } 1}, l i x i s t\right) \&$

$O b j \operatorname{cct}\left(* c_{\text {copy } 1}, * \operatorname{cop} y_{1}\right) \&$

CopyOf $\left(* \operatorname{cop} y_{1}, * o b j_{1}\right) \&$

$\operatorname{Loc}\left(* e_{\operatorname{cop} y 1}, * l_{\operatorname{cop} y 1}\right) \&$

Owner $\left(* l_{\text {copy } 1}, * r e c p_{1}\right) \&$

Time $\left(* e_{\text {cop } y 1}, * t_{\text {cop } y 1}\right)$.

(r16)TRel(l)uring, $\left.* t_{j 1}, * t_{o b j 1}\right)$.

(r17)TRel(Before, $\left.* t_{f 1}, * t_{\text {cop } y 1}\right)$.

In $(\mathrm{r} 1), * a g e n t_{1}, * r e c p_{1}$ and $* o b j_{1}$ represent respectively the pronominalized agent, recipiont, and object of forwarding. Variable $*$ recp $p_{1}$ is identified with IBill by unifying Hearer(*recp $p_{1}$ ) in (r1) with (r:12) in the context.

Representation (r14) is unified with $(\mathrm{r} 10),{ }^{10}$ and then $* a g e n t_{1}$ is identified with John and $* o b j_{1}$ is identified with $M$. Likewise, $* e_{o b j 1}, * l_{o b j 1}$, and $* l_{o b j 1}$ are identified respectively with $L_{m}^{\prime}, L_{m}$ and $T_{m}$.

Consequently, nominal reference in sentence (s4) is resolved. $\Lambda$ s stated below, identifying $* o b j_{1}$ with $M$ serves to resolve temporal reference.

By using ( $r 13$ ), variables, $* t_{s p 1}$ and $* t_{\text {focus } 1}$ are identified respectively with $T_{s p}$ and $T_{d}^{\prime}$.

The following temporal relationship is clerived from ( $\mathrm{r} 16$ ) because $* o b j_{1}$ is identified with $M$ and then $* t_{o b j 1}$ is identified with $I_{m}^{\prime}$.

(r18)THel( During, $\left.* \iota_{f 1}, T_{m}\right)$.

The following temporal relationship is derived from (rl) because $* t_{f o c u s}$ is identified with $l_{d}$.

(r19)TRel $\left(* v e l_{1}, * t_{f 1}, T_{d}\right)$.

Resolving temporal reference here means determining temporal relationship $* r e l_{1}$ in $(\mathrm{r} 19)$.

By default interpretation rule (r4), the following representation is derived from ( $\mathrm{r} 19)$.

(r20)TRel(After, $\left.* l_{f}, I_{d}\right)$.

On the other hand, by applying theorem ( $r(0)$ to (r19) and then using (r18) and (r11), the following representation is derived.

\section{(r21) T Rel ( Be fore, $\left.* l_{j 1}, T_{d}\right)$.}

Knowledge based interpretation (r21) is prelerred over default interpretation (120). Consequently, tho relcvant, temporal relationship between forwarding and deleting is determined.

linally, new constants $l_{j}^{r}, I_{j}, I_{\text {copy }},\left(C_{0} y, I_{\text {coopy }}\right.$, and $T_{\text {copy }}$ are generated for respectively variables $* e_{f 1}$, $* t_{f 1}, * e_{c o p y} 1, * c o p y_{1}, * l_{c o p y} 1$, and $* t_{c o p y 1}$. Constant Copy represents the copy of the e-mail message $M$, generated by forwarding $M$. Finally, the following representations are introduced into the context.

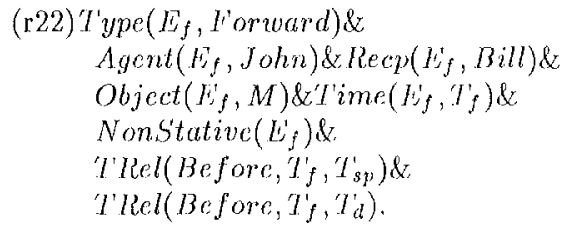

\footnotetext{
${ }^{10}$ Thepresentation $(915)$ cannot be unified with ( 110$)$ sinco $*_{\text {recp }}$ in (r15) is identified with Bill.
}

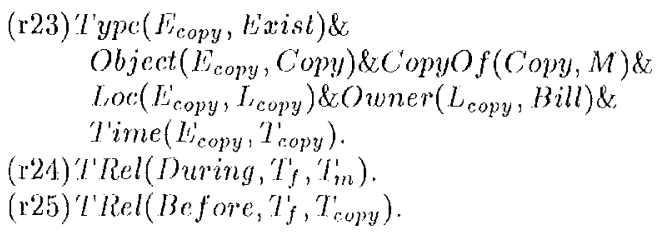

Next, consider the interpretation of sentence ( $s b$ ), which describes the following eventuality, $* e_{x 22}$.

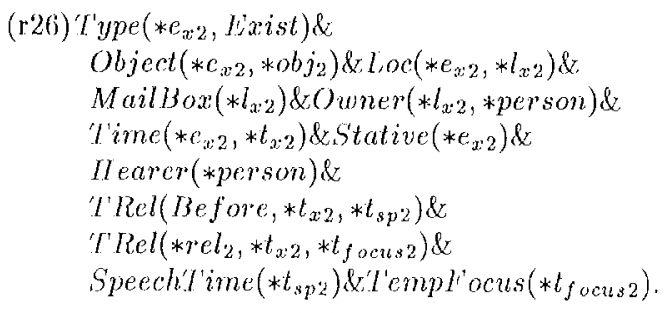

Ifere, I assumo that (s5) directly describes the existential status of an individual $* o b j_{2}$, which is realized as the zero-pronominalized subject in the sentence, and that the existential status is construed as a stative eventuality. 'The location, $* l_{n 2}$, of $* o b j_{2}$ is specified as a mail box that the hearer owns since the location is designated by "sochira no mail box (your mail box)". The tinne, $* t_{x 2}$, when $* o b j_{2}$ exists is before the speech time since the past-tense morpheme "ta" is used.

'Lhe referent for $* o b j_{2}$ is ambiguous since the refercnt can be either e-mail message $M$ or its copy $C o p y$. 'The relovant referent must be Copy. As argued below, resolving this nominal reference is crucial in resolving temporal reference.

I assume that the time of forwarding, ' $]_{f}^{\prime}$, is in the temporal focus. Resolving temporal reference here means determining temporal relationship * * el $l_{2}$ between $* l_{x 2}$ and $T_{f}$. 'I'he relevant interpretation is that $* l_{x 2}$ is after ' $l^{\prime} f$.

let ne explain how the framework interprets ( 85 ). First of all, *person in (r26) is identified with Bill, since *person mist be the hearer. 'Then $* e_{x 2}$ in $\left(\mathrm{r}^{2} 66^{2}\right)$ is iclentified with $L_{c o p}^{\dagger}$ in (123). Likewise, $* o b j_{2}, * l_{x 2}$, and $* l_{x 2}$ are identified respectively with $C o p y, l_{c o v y}$, and ' $T_{\text {copy }}$. 'Thus the zero-ptonominalized subject is appropriately interpreted. $\Lambda$ s a result, the following temporal relationship is derived from $(126)$ since $* l_{x 2}$ is identified with ' $l_{\text {copy } y}$ and $\eta_{f}^{\prime}$ is in the temporal focus.

$$
\text { (r'27) Thel } \operatorname{le}\left(* r_{2} l_{2}, T_{c o p y}^{\prime}, T_{f}^{\prime}\right) \text {. }
$$

Here, consider how the temporal relationship *rel, is determined. By using default interpretation rule $(15), * r c l_{2}$ is identificd with a relation, Contains, since the eventuality described by(s5) is stative.

On the other hand, by using ( 125$)$ and theorem (r.7), $* \mathrm{rel}_{2}$ is identified with a relation, $A f t e r$. In other words, the time of the eventuality described by (s5) follows the time of forwarding. 'Ithis knowledgebased interpretation is preferred over the defanlt interpretation, and is the relevant interpretation.

In the above process, identifying the pronominalized subject, $* o b j_{2}$, of ( 55 ) with Copy is crucial for the temporal reference resolution. Assume that $* o b j_{2}$ 
is identified with the e-mail message $M$. This case happens when $* e_{x 2}$ in ( $\left.\mathbf{r 2 6}\right)$ is identified with $E_{m}$ in (r10) and $* t_{x 2}$ is identified with $T_{m}$. In this case, the following temporal relationship is derived from (r26).

(r28)TRel $\left(* r e l_{2}, T_{m}, T_{f}\right)$.

By using ( $\mathrm{r} 24)$ and theorem ( $\mathrm{r} 8)$, $* r e l_{2}$ is identified with a relation, Contains. This interpretation is not relevant.

However, the case that leads to the wrong interpretation never occurs. When $* e_{x 2}$ is identified with $E_{m}$, the following representation is derived from (r26) since *person is identified with Bill.

$$
\text { (r29) Loc }\left(E_{m}, * l_{x 2}\right) \& \text { Owner }\left(* l_{x 2}, B i l l\right) \text {. }
$$

This representation is inconsistent with ( $r 10)$ since the owner of the location of the e-mail message $M$ must be uniquely identified. Thus, $* e_{x 2}$ must be identified with $E_{\text {copy }}$ and then $* o b j_{2}$ must be identified with Copy.

Consequently, identifying appropriately the referent of the pronominalized subject of sentence (s5) affects the process of resolving temporal reference, and this reference interaction can be explained by exploiting knowledge such as ( $\mathrm{r} 2)$ and (r3).

\section{CONCLUSION}

This paper has made a twofold contribution to research on temporal reference resolution. First, an account of the role played by the reference interaction in resolving temporal reference is given. As stated in sec. 2, existing models cannot account for how the process of resolving nominal reference affects the process of resolving temporal reference since they do not explain the reference interaction. Second, it is shown that exploiting the reference interaction clarifies how a restricted set of world knowledge (knowledge of the temporal relationships between eventualities and the existential status of individuals) contributes to the resolution process. As stated in sec. 2, existing models resort to the entire set of causality knowledge. I do not entirely deny the validity of the existing approach. However, when a restricted set of world knowledge is sufficient to resolve temporal reference, immediate recourse to the entire set of world knowledge of causality can be avoided. A framework is also presented, within which temporal reference is resolved by exploiting the reference interaction.

Future work will first extend the framework to deal with eventualities specified by various tense morphemes, whereas this paper focuses on eventualities specified by the past-tense morpheme "ta". Next, to explore the notion of reference interaction in more detail, the way resolving temporal reference affects the resolution of nominal reference must be investigated, whereas this paper concentrates on the converse process. Finally, the coverage of this framework must be evaluated. Evaluation will require an exhaustive listing of possible cases of the existential status of individuals that are used to resolve reference. This paper accounts for cases where an individual exists at a certain space-time location of the physical world. We can allow for other kinds of existential status, such as a status where an individual exists in the mental state of a dialogue participant. ${ }^{11}$

\section{REFERENCE}

Allen, J. F. (1983). Maintaining knowledge about temporal intervals. Communication of the ACM, 26, 832-843.

Bach, E. (1986). The algebra of cvents. Linguistics and Philosophy, 9, 5-16.

Brent, M. R. (1990). A simplified theory of tense representations and constraints on their composition. In: Proc. of the 28th Annual Meeting of the Association for Computational Linguistics, 119-126.

Crouch, R. S. and S. G. Pulman (1993). Time and modality in a natural language interface to a planning system. Artificial Intelligence, 63, 265-304.

Davidson, D. (1980). Lssays on Actions and Events. Oxford University Press.

Grosz, B. J., A. K. Joshi and S. Weinstein (1983). Providing a unified account of definite noun phrase in discourse. In: Proc. of the 21th Annual Meetings of the Association for Computational Linguistics, 44-49.

Hinrichs, E. (1986). Temporal anaphora in discourses of English. Linguistic and Philosophy, 9, 63-82.

Hirst, G. (1991). Existence assumptions in knowledge representation. Artificial Intelligence, 49, 199-242.

Hwang, C. H. and I. K. Schubert (1992). Tense trees as the "fine structure" of discourse. In: Proc. of the 30th Annual Meeting of the Association for Computational Singuistics, 232-240.

Kameyama, M. (1986). A property-sharing constraint in centering. In: Proc of the 24th Annual Meetings of the Association for Computational Linguistics, 200-206.

Kameyama, M., R. Passonnean and M. Poesio (1993). Temporal centering. In: Proc. of the 31th Annual Meeting of the Association for Computational Linguistics, $70-77$

Lascarides, A. (1992). Knowledge, causality, and temporal representation. Linguistics, 30, 941-973.

Partee, B. H. (1984). Nominal and temporal anaphora. Linguistics and Philosophy, 7, 243-286.

Reichenbach, H. (1947). Elements of Symbolic Logic. Macmillan, New York.

Song, F. and R. Cohen (1991). 'Tense interpretation in the context of narrative. In: Proc. of the 9th National Conference on Artificial Intelligence, 131-136.

Vlach, F. (1993). Temporal adverbials, tenses and the perfect. Linguistics and Philosophy, 16, 231-283.

Webber, B. L. (1988). Tense as discourse anaphor. Computational Linguistics, 14, 61-73.

Webber, B. L. and B. Baldwin (1992). Accommodating context change. In: Proc. of the 90th Annual Meeting of the Association for Computational Linguistics, 96103

\footnotetext{
${ }^{11}$ Various kinds of existence are presented by Hirst (1991).
} 